\title{
Parameter estimation of an aeroelastic aircraft using neural networks
}

\author{
S C RAISINGHANI and A K GHOSH
}

Department of Aerospace Engineering, Indian Institute of Technology Kanpur, Kanpur 208 016, India

e-mail: scr@iitk.ac.in

\begin{abstract}
Application of neural networks to the problem of aerodynamic modelling and parameter estimation for aeroelastic aircraft is addressed. A neural model capable of predicting generalized force and moment coefficients using measured motion and control variables only, without any need for conventional normal elastic variables or their time derivatives, is proposed. Furthermore, it is shown that such a neural model can be used to extract equivalent stability and control derivatives of a flexible aircraft. Results are presented for aircraft with different levels of flexibility to demonstrate the utility of the neural approach for both modelling and estimation of parameters.
\end{abstract}

Keywords. Parameter estimation; modelling; aeroelastic aircraft; neural networks; system identification.

\section{Introduction}

Applications of the parameter estimation method to extract aircraft parameters (stability and control derivatives) from flight data in the linear flight regime for rigid aircraft have been highly successful in the past (Maine \& Iliff 1986). However, the newly introduced highly maneuverable aircraft with their high degree of flexibility pose a new challenge to search for appropriate aeroelastic models for inclusion in parameter estimation algorithms (Zerweckh et al 1990). One of the basic problems faced in estimating parameters of an aeroelastic aircraft is: a full order model of an aeroelastic aircraft has an order of magnitude larger number of parameters, and thus, too many of them yield satisfactory estimates via any of the known parameter estimation methods. To simplify dynamic analysis and control synthesis, or to ease computational burden in simulation, simple low order models of vehicle dynamics have been proposed by Waszak \& Schmidt (1988) and Ghosh \& Raisinghani (1993). Many proposed model reduction procedures rely on numerical techniques and/or transformations, and thereby, in the resulting models, the physics of the system is far from transparent (Waszak \& Schmidt 1988). Ghosh \& Raisinghani (1993) proposed a reduced order model of an aeroelastic aircraft that contains the essential characteristics of aircraft dynamics, and also retains and provides physical interpretation of the parameters involved in the reduced order model. Furthermore, they have also given a criterion in the form of an 
analytical expression to help decide adequacy or otherwise of the proposed simplified model of an aeroelastic aircraft. In the present work, rather than resorting to model simplification for estimating parameters, we use a neural network aerodynamic model of a flexible aircraft, and subsequently use such a model for estimating parameters via the Delta and the Zero methods (discussed later) proposed by Raisinghani et al (1998a).

Recently, a class of neural networks called the feed forward neural networks (FFNNs) have been used to model aircraft dynamics wherein aircraft motion variables and control inputs are mapped to predict the total aerodynamic coefficients (Hess 1993; Basappa \& Jategaonkar 1995; Linse \& Stengal 1993). It has been shown that FFNNs can work as general function approximators and thereby are capable of approximating any continuous function to any desired accuracy provided the appropriate number of hidden layers and the neurons per layer exist, and that the activation function is continuous (Hornik et al 1989). The capacity to act as general function approximator presents FFNNs as an alternative tool for modelling aircraft dynamics. FFNNs also possess generalization property which enables the interpolation and extrapolation using a finite set of measurements. This generalization property has been exploited by Raisinghani et al (1998a) to propose two methods, named the Delta method and the Zero method, for explicitly estimating aircraft parameters using FFNNs. This paper investigates the aerodynamic modelling of an aeroelastic aircraft using FFNN and the applicability of the Delta and Zero methods for extracting parameters from such a neural model.

\section{Neural networks}

Neural networks have two main components - the processing elements called neurons or nodes and the connection between neurons, each connection having its own weight. The neurons are the information processors, and the connections function as information storage (figure 1a). Each processing element first calculates a weighted sum of the input signals, and then applies a transfer function, also called activation function, such as the tangent hyperbolic function or the logistic (sigmoidal) function to the weighted sum and outputs the result. The neurons within a network are arranged in an input layer, one or more hidden or processing layers, and an output layer. Figure $1 \mathrm{~b}$ shows a typical backpropagation neural network. The name 'backpropagation' comes from the training method employed during the learning (training) process - backpropagation of error. The error between the predicted output and expected (desired or true) output is computed. The training method is simply a gradient descent method that minimizes the total squared error of the output so computed by the net. In a prediction problem, such as aerodynamic modelling, the number of neurons in the input layer equals the number of input variables, and the number of neurons in the output layer equals the number of predicted variables. Selection of the rest of the architecture of the network in terms of the number of neurons in the hidden layer, the learning rate, the momentum rate etc. is not an exact science, and one has to resort to trial and error methods to find a suitable network structure for the given data.

\section{The Delta method and the Zero method}

Details of both the Delta and Zero methods are given in Raisinghani et al (1998) and Ghosh et al (1998). However, for the sake of completeness, a brief description of the methods is presented. The Delta method is based on the following premise: a stability or a control 


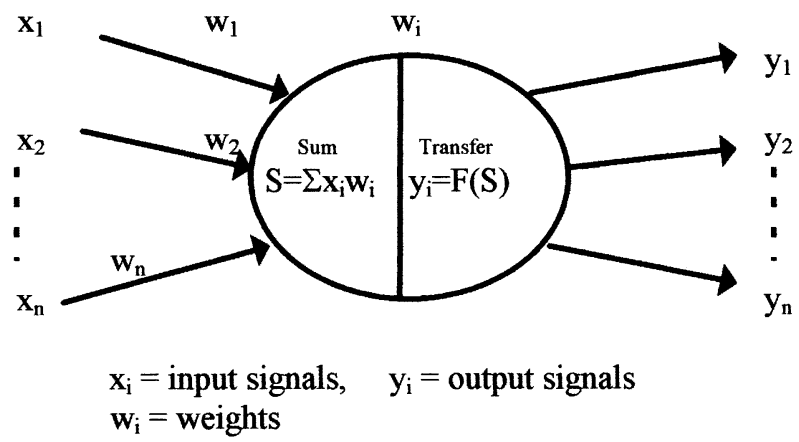

(a)

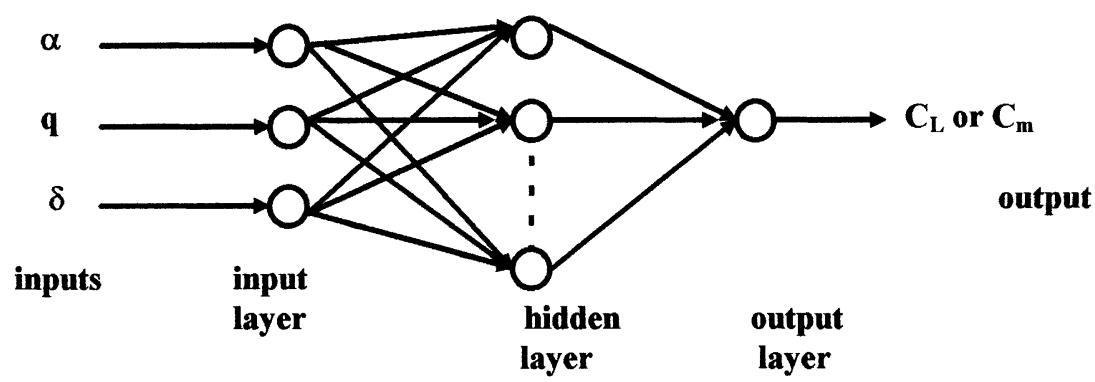

(b)

Figure 1. Schematic of (a) a processing element, (b) a backpropagation neural network for modelling short period mode.

derivative can be thought of as the change in the aerodynamic force or moment coefficient caused by a small variation in one of the motion/control variables while the rest of the variables are held constant. Let the FFNN be trained to map the network input variables $\alpha$, $q$ and $\delta$ to the output variable $C_{L}$ or $C_{m}$. Only one of the network inputs (say $\alpha$ ) is given a small (delta, $\pm \Delta$ ) perturbation (hence the name "the Delta method") at each time point while all the other network inputs are held at their original values. Let the predicted $C_{m}$ for $\alpha+\Delta \alpha$ and $\alpha-\Delta \alpha$ be $C_{m}^{+}$and $C_{m}^{-}$. Now the stability derivative $C_{m_{\alpha}}$ is given by $C_{m_{\alpha}}=$ $\left(C_{m}^{+}-C_{m}^{-}\right) / 2 \Delta \alpha$.

The Zero method views the stability or control derivatives as follows: if we could obtain an aerodynamic coefficient as a function of only one of the motion/control variables at a time, while the rest of the variables are set at zero values, then the ratio of the predicted aerodynamic coefficient to the non-zero motion/control variable will yield the corresponding stability/control derivative. For example, if we set $q=\delta=0$ while $\alpha$ is kept at its original value, the predicted $C_{L}$ and $C_{m}$ are used to yield $C_{L_{\alpha}}=C_{L} / \alpha$ and $C_{m_{\alpha}}=C_{m} / \alpha$.

\section{Simulated flight data generation and neural modelling}

At the outset, it may be emphasized that the Delta and Zero methods do not require stipulation of an a priori model for flexible aircraft; it is only for the purpose of generating 
simulated flight data that the following equations of motion are considered. To this purpose, only the longitudinal nonlinear equations of motion of a flexible aircraft are considered and linearized about a straight and level cruise flight. Assuming variations in velocity to be negligible $(u=$ constant), the equation of motion given by Waszak \& Schmidt (1988) are approximated for the short period mode as

$$
\begin{aligned}
\dot{\alpha}-q & =-\rho u S / 2 m\left[C_{L \alpha} \alpha+C_{L q} q c / 2 u+C_{L \delta} \delta+\sum_{i=1}^{n}\left(C_{L n i} n_{i}+C_{L \dot{n} i} \dot{n}_{i} c / 2 u\right)\right], \\
\dot{q} & =\rho u^{2} S c / 2 I_{y}\left[C_{m \alpha} \alpha+C_{m q} q c / 2 u+C_{m \delta} \delta+\sum_{i=1}^{n}\left(C_{m n i} n_{i}+C_{m i n} \dot{n}_{i} c / 2 u\right)\right],
\end{aligned}
$$

where vehicle angle-of-attack $\alpha$, pitch rate $q$, and control input $\delta$ represent small perturbations from the chosen reference flight condition; $n_{i}$ and $\dot{n}_{i}$ are the generalized elastic deflections and their time derivatives; $C_{L \alpha}, C_{m \delta}, \ldots, C_{L n i}, C_{m n i}$ are the stability and control derivatives as defined in Waszak \& Schmidt (1988). Air density $\rho$, total inertial velocity $u$, wing area $S$, wing chord $c$, aircraft mass $m$, and moment of inertia about the $Y$-axis $I_{y}$, are the other quantities used in the above equations.

The following equation satisfied by the generalized coordinates $n_{i}$ is taken from Waszak $\&$ Schmidt (1988) except that an additional term $2 \xi_{i} w_{i} \dot{n}_{i}$ representing the structural damping is also included in it

$$
\begin{aligned}
\ddot{n}_{i} & +2 \xi_{i} w_{i} \dot{n}_{i}+w_{i}^{2} n_{i}=\rho u^{2} S c / 2 M_{i}\left[C_{\alpha}^{n i} \alpha+C_{q}^{n i} q c / 2 u+C_{\delta}^{n i} \delta\right. \\
& \left.+\sum_{j=1}^{n}\left(C_{n j}^{n i} n_{j}+C_{\dot{n} j}^{n i} \dot{n}_{j} c / 2 u\right)\right],
\end{aligned}
$$

where $w_{i}, \xi_{i}$ and $M_{i}$ are the in-vacuo frequency, modal damping, and modal generalized mass respectively and $C_{\alpha}^{n i}, C_{q}^{n i}, \ldots, C_{n i}^{n i}, C_{n i}^{n i}$ represent the generalized force derivatives due to coupling in elastic and aerodynamic degrees-of-freedom.

Due to nonavailability of real flight data, simulated flight data were generated for an example aeroelastic aircraft similar to that given in Waszak \& Schmidt (1988). The geometric, mass, and moment of inertia characteristics, stability and control derivatives, flight condition, and the first four aeroelastic modes for the baseline configuration $\mathrm{C} 2$ and its more flexible version C3 are taken from Waszak \& Schmidt (1988). Equations (1)-(2) are integrated for a multistep 3-2-1-1 type of elevator input to generate motion variables $(\alpha, q)$ and to compute generalized force and moment coefficients $\left(C_{L}\right.$ and $\left.C_{m}\right)$. To vary the flexibility of the aircraft, it is assumed that the aircraft can be made to vibrate in nearly the same normal modes but with different in-vacuo frequencies (Waszak \& Schmidt 1988). Thus, for the more flexible configuration $\mathrm{C} 3$, the same total force coefficients as that of $\mathrm{C} 2$ - but the in-vacuo frequencies of $\mathrm{C} 3$ - were used to generate flight data. Now the motion and control variables $\alpha, q$ and $\delta$ as the network inputs and either $C_{L}$ or $C_{m}$ as the output variable are used for supervised training of the network.

The training algorithm is iterative in nature: the algorithm is started with a set of randomly initialized weights, and the back propagation algorithm repeated for all data points to update the weights recursively. The mean square error (MSE) for each iteration is defined by,

$$
\text { MSE }=\frac{1}{m n} \sum_{i=1}^{n} \sum_{j=1}^{m}\left[Y_{i}(j)-X_{i}(j)\right]^{2}
$$


where $Y$ and $X$ are respectively the desired and computed outputs of the neural network, $n$ is the number of data points and $m$ is the number of output variables. The training sessions are continued till changes in the MSE in successive iterations are less than the prescribed value, or the number of iterations exceeds the specified number.

Once the training (modelling) is over and the network weights are frozen, the same input data are passed again to check the prediction capability of the neural network. The predicted values are deemed acceptable only if the MSE is less than the minimum specified value. To achieve the desired level of MSE, various interrelated neural network parameters like the number of hidden layers, number of nodes in the hidden layer(s), learning rate, momentum factor, abruptness (gain) factor of the sigmoidal function, initial neural network weights and scaling of input-output data are adjusted by trial and error. Once the FFNN has been trained satisfactorily to represent the mapping of the input variable $(\alpha, q, \delta)$ to the output variable $\left(C_{L}\right.$ or $\left.C_{m}\right)$, it is used for estimating aircraft parameters by the Delta and Zero methods.

The trained FFNN is the aerodynamic model of the aeroelastic aircraft, mapping motion and control variable to the total force and moment coefficient. Such an FFNN aerodynamic model has few useful practical applications besides its use for estimating parameters via the Delta and Zero methods. For example, such an on-board neural model could provide access to an on-line aeroelastic model of an aeroelastic aircraft; the model would predict the total aerodynamic force and moment coefficients (including the elastic effects), the measured variables required being only the motion and control variables, and not the elastic deflections or their time derivatives. In this context, it is worth reiterating that for the case of real flight data, only the measured variables $\alpha, q, \delta$, normal acceleration $a_{z}$ and $\dot{q}$ (either measured directly, or obtained via numerical differentiation of measured $q$ ) are required. The total aerodynamic coefficients (with aeroelastic effects included) $C_{L}$ and $C_{m}$ are computed from $C_{L}=-2 m a_{z} / \rho u^{2} S$ and $C_{m}=I_{y} \dot{q} / \rho u^{2} S c$.

Thus, the equations of motion, (1) and (2), neither need to be postulated nor integrated for neural modelling, i.e., the neural model is obtainable by directly using only the measured quantities.

\section{Parameter estimation via the Delta and the Zero methods}

The Delta and Zero methods possess some features that could be used advantageously when estimating parameters of an aeroelastic aircraft. Because the network is trained separately for each of the force or moment coefficients, it can be subsequently used for estimating parameters, one at a time, corresponding to that very force or moment coefficient. Thus, rather than estimating the whole set of parameters in one go, the estimation is carried out in separate steps equal to the number of force or moment coefficients involved. For example, the stability and control derivatives corresponding to each of the six degrees-of-freedom are obtained separately. This property is of special significance for an aeroelastic aircraft since the number of unknown parameters is very large. Furthermore, for an aeroelastic aircraft, due to elastic deformations, the rigid-body and elastic degrees-of-freedom get coupled via the aerodynamic forces. Conventional methods like the maximum likelihood (ML) method would require solution of these coupled equations to generate the model response which is compared with the measured response to form the cost function to be minimized by the ML algorithm. Also, the ML method needs a reasonable estimate for initial values of parameters to begin the algorithm. In contrast, the Delta and the Zero 
Table 1. Comparison of parameter estimates via the Delta and Zero methods, and analytical computations.

\begin{tabular}{|c|c|c|c|c|c|c|}
\hline \multirow[b]{3}{*}{ Parameter } & \multicolumn{6}{|c|}{ Estimated equivalent parameters } \\
\hline & \multicolumn{3}{|c|}{ Configuration $\mathrm{C} 2$} & \multicolumn{3}{|c|}{ Configuration $\mathrm{C} 3$} \\
\hline & Delta & Zero & Analytical & Delta & Zero & Analytical \\
\hline$C_{L \alpha}$ & $\begin{array}{c}2.629 \\
(0.076) *\end{array}$ & $\begin{array}{c}2.662 \\
(0.017)\end{array}$ & 2.700 & $\begin{array}{r}1.997 \\
(0.59)\end{array}$ & $\begin{array}{l}2.433 \\
(0.25)\end{array}$ & 2.24 \\
\hline$-C_{L q}$ & $\begin{array}{l}15.286 \\
(0.426)\end{array}$ & $\begin{array}{l}14.817 \\
(0.091)\end{array}$ & 15.870 & $\begin{array}{l}15.238 \\
(5.00)\end{array}$ & $\begin{array}{c}21.473 \\
(0.111)\end{array}$ & 18.57 \\
\hline$C_{L \delta}$ & $\begin{array}{c}0.367 \\
(0.015)\end{array}$ & $\begin{array}{c}0.374 \\
(0.007)\end{array}$ & 0.351 & $\begin{array}{c}0.203 \\
(0.10)\end{array}$ & $\begin{array}{c}0.317 \\
(0.005)\end{array}$ & 0.021 \\
\hline$-C_{m \alpha}$ & $\begin{array}{c}1.287 \\
(0.060)\end{array}$ & $\begin{array}{c}1.346 \\
(0.018)\end{array}$ & 1.390 & $\begin{array}{c}0.544 \\
(0.08)\end{array}$ & $\begin{array}{c}0.598 \\
(0.01)\end{array}$ & 1.565 \\
\hline$-C_{m q}$ & $\begin{array}{l}36.358 \\
(1.547)\end{array}$ & $\begin{array}{l}37.646 \\
(1.166)\end{array}$ & 33.100 & $\begin{array}{l}18.803 \\
(2.93)\end{array}$ & $\begin{array}{l}20.773 \\
(1.46)\end{array}$ & 27.98 \\
\hline$-C_{m \delta}$ & $\begin{array}{c}2.331 \\
(0.097)\end{array}$ & $\begin{array}{c}2.311 \\
(0.193)\end{array}$ & 2.310 & $\begin{array}{l}1.385 \\
(0.22)\end{array}$ & $\begin{array}{l}1.502 \\
(0.02)\end{array}$ & 1.573 \\
\hline
\end{tabular}

* Sample standard deviation in parentheses

methods require neither the solution of such coupled equations nor any guess values of the parameters.

We reiterate: it is only for the sole purpose of generating simulated flight data that the coupled equations of motion (1) and (2) are required, and not for estimating parameters via the Delta or the Zero method. The flight data so generated always contain the aeroelastic effects of all the four modes, and thus the generalized force and moment coefficients in the output file of the network always contain all the aeroelastic effects. However, the variables used in the network input file can be varied. In the present work, the results for the following two cases are presented.

Case 1: The network input file has only the motion and control variables $\alpha, q$ and $\delta$. None of the elastic deflections or their derivatives are present in the input file.

Case 2: The input file variables $\alpha, q$ and $\delta$ are supplemented by the elastic deflection $n_{1}$ corresponding to the first mode.

\subsection{Results for case 1}

The aerodynamic model for case 1 achieves mapping of the motion and control variables to the total force and moment coefficients that contain the aeroelastic effects. The estimated parameters via the Delta and the Zero methods from such a neural model (table 1) would represent equivalent parameters wherein the effects of all the aeroelastic modes get absorbed. Before discussing these results, we digress to place the concept of equivalent derivatives in its proper perspective.

A question is posed: When and how useful are the equivalent parameter estimates? Ghosh \& Raisinghani (1993) proposed an analytical expression $F$ as an indicator of the flexibility of the aircraft and of its adequacy or otherwise of using equivalent parameters to represent essential dynamics of the flexible aircraft. The analytical expression $F$ is obtained 
Table 2. Effect of inclusion of mode shapes $\left(n_{i}, i=1,2,3\right)$ on computed equivalent parameters.

\begin{tabular}{lcccccccc}
\hline & \multicolumn{9}{c}{ Analytically computed equivalent parameters } \\
\cline { 3 - 5 } & Rigid & \multicolumn{3}{c}{ Configuration C2 } & & \multicolumn{3}{c}{ Configuration C3 } \\
\cline { 3 - 5 } \cline { 7 - 9 } Parameter & body & $n 1^{*}$ & $n 1, n 2^{\#}$ & $n 1, n 2, n 3^{\$}$ & & $n 1$ & $n 1, n 2$ & $n 1, n 2, n 3$ \\
\hline$C_{L \alpha}$ & 2.92 & 2.71 & 2.72 & 2.70 & & 1.995 & 2.26 & 2.24 \\
$-C_{L q}$ & 14.70 & 16.04 & 15.84 & 15.87 & & 8.79 & 18.56 & 18.57 \\
$C_{L \delta}$ & 0.435 & 0.252 & 0.375 & 0.351 & & -0.363 & 0.08 & 0.021 \\
$-C_{m \alpha}$ & 1.66 & 1.42 & 1.42 & 1.39 & & 0.63 & 0.610 & 0.565 \\
$-C_{m q}$ & 34.75 & 33.25 & 33.22 & 33.10 & & 28.17 & 28.04 & 27.98 \\
$-C_{m}$ & 2.57 & 2.37 & 2.363 & 2.31 & & 1.69 & 1.66 & 1.573 \\
\hline
\end{tabular}

* Only first ( $n 1)$ mode included; $\#$ only first $(n 1)$ and second $(n 2)$ modes included; ${ }^{\circledR}$ only first $(n 1)$, second $(n 2)$ and third (n3) modes included

by substituting steady state $n_{i}$ (dropping the time derivative terms $\ddot{n}_{i}, \dot{n}_{i}$ ) from (2) into (1). Such an analytical expression and the corresponding computed equivalent parameters for the case where only the first mode terms $\left(n_{1}\right)$ are retained in (2) are given by Ghosh \& Raisinghani (1993). It is of interest to see how the equivalent parameters are affected by inclusion of the second, or the second and third modes, in addition to the first mode included earlier. In table 2, analytically computed parameters from such an exercise are compared for the nominal configuration $\mathrm{C} 2$ and its more flexible version $\mathrm{C} 3$. Results for the $\mathrm{C} 2$ configuration show that the inclusion of the first mode alone is sufficient to predict the equivalent parameters, since inclusion of additional modes affects analytically computed values only marginally (except $C_{L \delta}$ ). For configuration C3, inclusion of additional modes does affect the estimates, the effect being most pronounced on the weak parameters $C_{L q}$ and $C_{L \delta}$. These observations suggest that for most of the strong parameters, inclusion of the first mode alone would be sufficient to analytically compute equivalent parameters.

The equivalent parameter estimates via the Delta and the Zero method would represent absorption of the all the aeroelastic modes. For comparison, table 1 lists these estimates along with the analytically computed values. For configuration $\mathrm{C} 2$, we see that these estimates compare reasonably with those obtained via analytical computations. For the more flexible configuration $\mathrm{C} 3$, the estimates show some discrepancy amongst those from different approaches, specially for the derivative $C_{L \delta}$.

The estimates via the Zero method show sample standard deviations lower by a factor of 2 to 10 as compared to the Delta method, and also compare marginally better with the analytically computed estimates. To illustrate how the aeroelastic effects would affect the aircraft response for a given control input, true $\alpha$ and $q$ responses for the more flexible configuration $\mathrm{C} 3$ are compared with the corresponding rigid-body responses in figure 2 . As seen, both $\alpha$ and $q$ responses for the aeroelastic aircraft are different, not only quantitatively but also qualitatively from the corresponding rigid-body response. Thus a rigidbody model for $\mathrm{C} 3$ would be erroneous and unacceptable. Next, the responses using estimated equivalent parameters from the Delta method, the Zero method and the analytical computations for $\mathrm{C} 3$ are compared with the actual response in figure 3. The estimated responses show a much improved match with the actual response; the match being better both quantitatively and qualitatively as compared to that shown for the rigid-body in figure 2. For configuration $\mathrm{C} 2$, the match (not shown) was found to be far better than that 
(a)

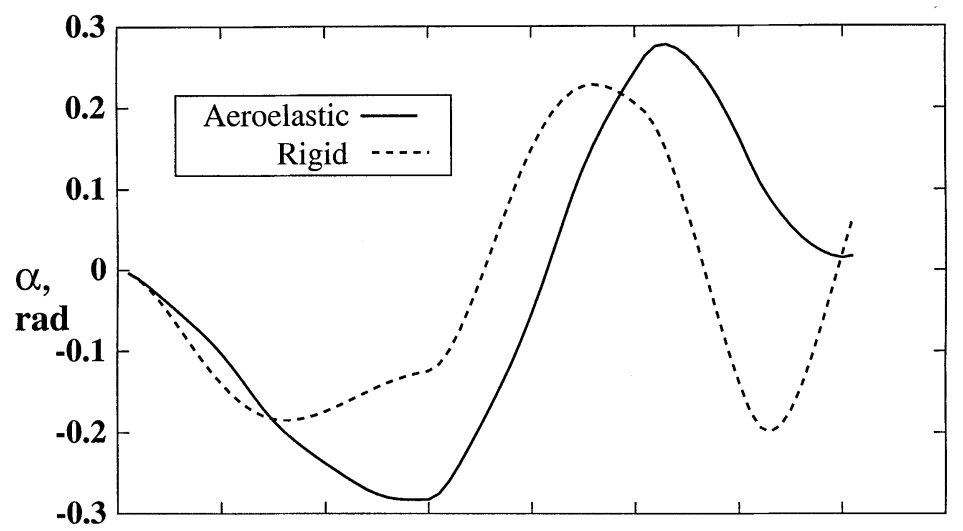

(b)

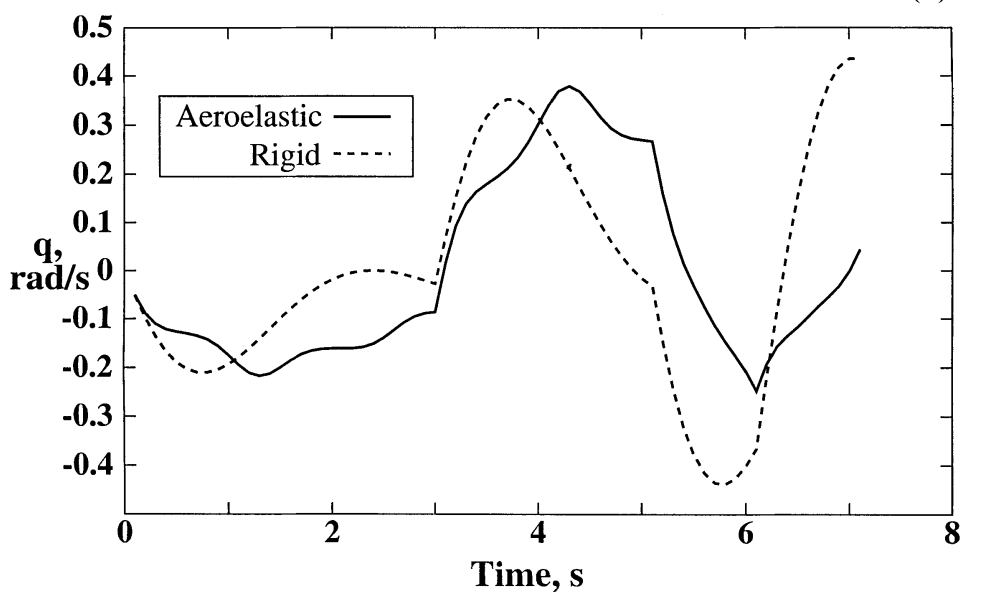

Figure 2. Comparison of responses when aeroelastic effects are omitted (rigid-body) and included for the aeroelastic configuration $\mathrm{C} 3$.

for $\mathrm{C} 3$. The above results suggest the potential use of the neural model, and the Delta or the Zero method for estimating parameters of an aeroelastic aircraft in design of control laws and flight simulators. A reasonable model as well as estimates of equivalent parameters are obtainable by simply using measured quantities without any need for postulating coupled equations of motion or guess for initial values of parameters to be estimated.

\subsection{Results for case 2}

For case 2, the input file used for aerodynamic modelling included the elastic deflection $n_{1}$ also. The idea is to see if the derivatives $C_{L n 1}$ and $C_{m n 1}$ could also be estimated. In addition to the equivalent parameters, reasonable estimates of $C_{L n 1}$ and $C_{m n 1}$ were obtained via the Delta and the Zero methods as shown below. Since the two additional parameters $C_{L n 1}$ and $C_{m n 1}$ to account for aeroelastic effects are added, the equivalent parameter values are closer to the rigid-body true values, specially for the $C_{m}$-derivatives as illustrated below. 
(a)

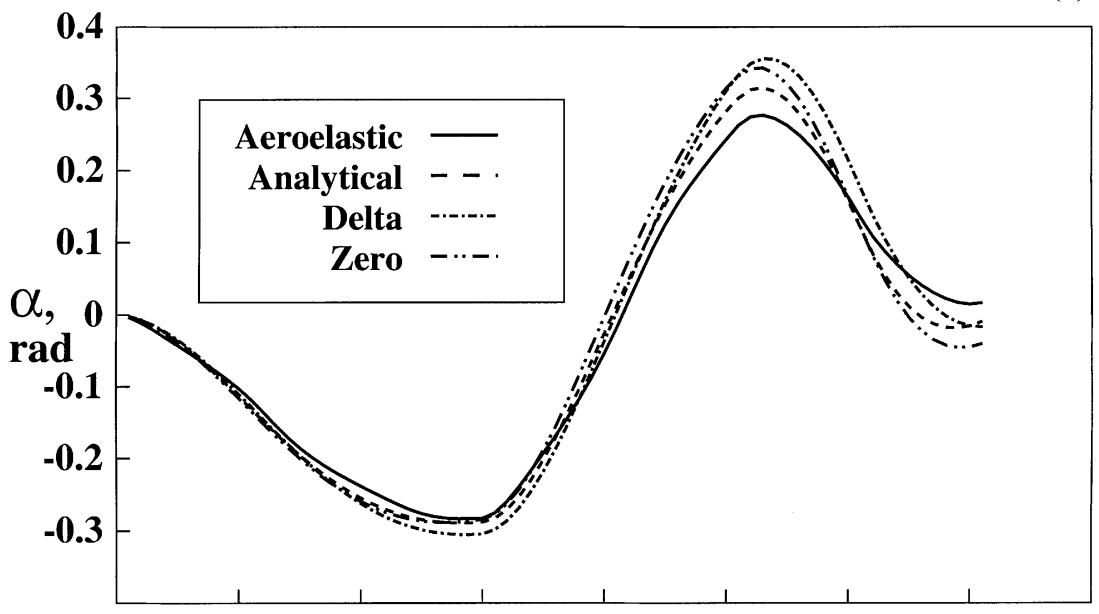

(b)

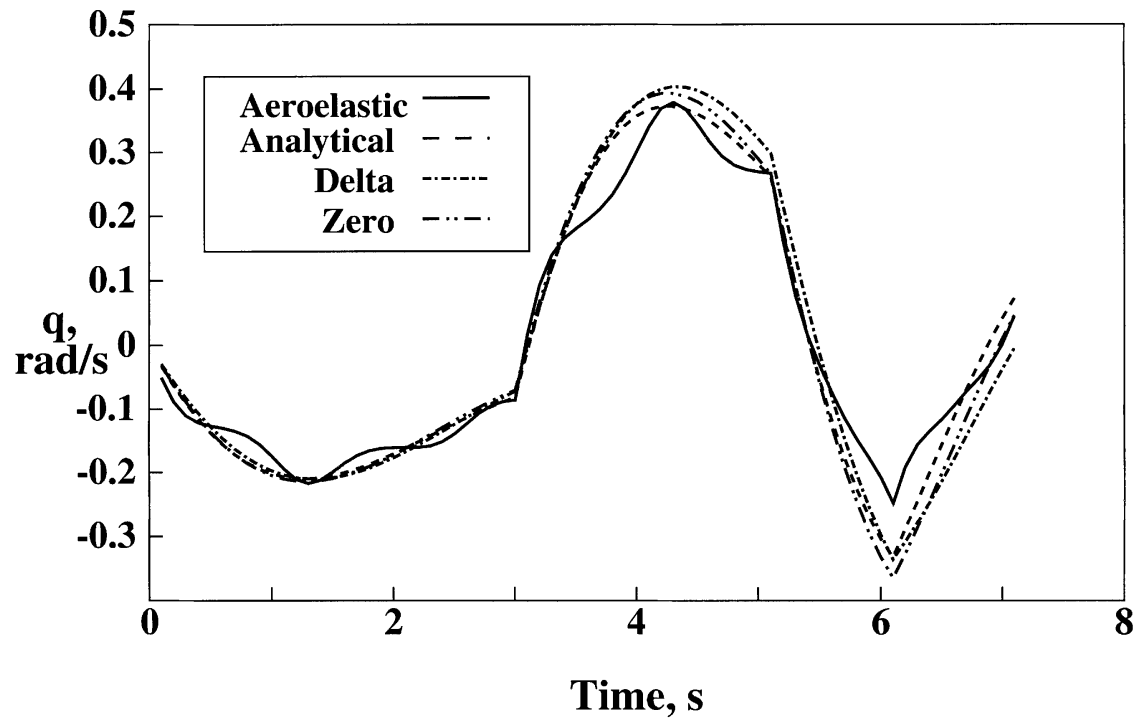

Figure 3. Comparison of actual responses of aircraft $\mathrm{C} 3$ with estimated responses using parameter estimates obtained via the Delta method, the Zero method and the analytical computations.

\section{True}

$C_{L n 1}$

$C_{m n 1}$

$C_{m \alpha}$

$C_{m q}$

$C_{m \delta}$$$
-34.75
$$$$
-2.57
$$

Delta

$$
\begin{gathered}
0.020(0.008)^{*} \\
-0.026(0.004) \\
-1.514(0.206) \\
-34.54(5.53) \\
-2.53(0.321)
\end{gathered}
$$

Zero

$$
\begin{aligned}
0.033(0.0001) \\
-0.028(0.001) \\
-1.612(0.055) \\
-37.85(3.66) \\
-2.67(0.328)
\end{aligned}
$$

* Sample standard deviation 


\section{Conclusions}

The results of applying the Delta and Zero methods to extract equivalent stability and control derivatives of an aeroelastic aircraft have been presented. It has been shown that the neural model of an aeroelastic aircraft can be achieved simply by using measured quantities $\alpha, q, \delta, a_{z}$ and $\dot{q}$ (obtain directly or via the numerical differentiation of measured $q$ ), without requiring measurements of elastic deflections or their derivatives. The main advantages of the neural modelling and estimating parameters from such a model via the Delta and Zero methods are: (i) it circumvents the need for postulating and solving the coupled rigid-body and elastic degrees-of-freedom equations for an aeroelastic aircraft, (ii) the problem of large numbers of parameters for an aeroelastic aircraft is reduced to estimating fewer parameters in terms of equivalent parameters and also, the parameters corresponding to each one of the degrees-of-freedom is estimated separately, (iii) no guesses of initial values of parameters are required by the Delta or Zero methods, and (iv) the on-board trained neural model has a potential for obtaining on-line parameter estimates, and such a model and/or parameter estimates can be used for flight control system design. A question may be posed: for on-line modelling and parameter estimation, the measurement errors due to bias, scale factor and measurement noise in the measured data would probably require on-line pre-processing of data. However, in a recent work by Raisinghani et al (1999), it has been shown that the proposed neural approach for modelling and parameter estimation can handle the raw data without a priori burden of correcting them for the measurement errors.

We would like to thank Dr P K Kalra of the Electrical Engineering Department for some useful discussions and the use of the neural simulator at his laboratory.

\section{References}

Basappa, Jategaonkar R V 1995 Aspects of feed forward neural network modelling and its application to lateral-directional flight data. DLR IB-111-95/30

Ghosh A K, Raisinghani S C 1993 Parameter estimates of an aeroelastic aircraft as affected by model simplifications. J. Aircraft 31: 452-454

Ghosh A K, Raisinghani S C, Khubchandani S 1998 Estimation of aircraft lateral-directional parameters using neural networks. J. Aircraft 35: 876-881

Hess R A 1993 On the use of backpropagation with feed forward neural networks for the aerodynamic estimation problem. AIAA paper no. 93-3639

Hornik K, Stinchcombe M, White H 1989 Multi layer feed forward neural networks are universal approximators. Neural Networks 2: 359-366

Linse D J, Stengal R F 1993 Identification of aerodynamic coefficients using computational neural networks. J. Guidance, Control Dyn. 16: 1018-1025

Maine R E, Iliff K W 1986 Identification of dynamic system-application to aircraft, Part I. Agard AG-300

Raisinghani S C, Ghosh A K, Kalra P K 1998a Two new techniques for aircraft parameter estimation using neural networks. Aeronaut. J. 102: 25-29

Raisinghani S C, Ghosh A K, Kalra P K 1998b Aircraft lateral-directional parameter estimation via neural networks. Proceedings of 49th AGM of the Aeronautical Society of India, Bangalore, pp 6.1-6.7 
Raisinghani S C, Rao C, Ghosh A K 1999 Effects of measurement errors on parameter estimation via neural networks. AIAA paper no. 99-4249

Waszak M R, Schmidt D K 1988 Flight dynamics of aeroelastic vehicles. J. Aircraft 25: 563-571

Zerweckh S H, Von Flotow A H, Murray J E 1990 Flight testing of highly flexible aircraft. J. Aircraft 27: $342-349$ 